\title{
Correction to: Infections in Infants with SCID: Isolation, Infection Screening and Prophylaxis in PIDTC Centers
}

\author{
Morna Dorsey $^{1}$ - Nicola A. M. Wright ${ }^{2} \cdot$ Natalia S. Chaimowitz $^{3}$ - Blachy J. Dávila Saldaña ${ }^{4,5} \cdot$ Holly Miller $^{6}$. \\ Michael D. Keller ${ }^{7}$. Monica S. Thakar ${ }^{8}$ - Ami J. Shah ${ }^{9} \cdot$ Rolla Abu-Arja $^{10}$ - Jeffrey Andolina ${ }^{11}$ • Victor Aquino ${ }^{12}$. \\ J. L. Barnum ${ }^{13}$. Jeffrey J. Bednarski ${ }^{14} \cdot$ Monica Bhatia ${ }^{15} \cdot$ Francisco A. Bonilla $^{16} \cdot$ Manish J. Butte $^{17} \cdot$ Nancy J. Bunin $^{18}$. \\ Lauri M. Burroughs ${ }^{8}$. Sharat Chandra ${ }^{19,20}$. Sonali Chaudhury ${ }^{21} \cdot$ Karin Chen $^{22}$. Hey Chong ${ }^{13}$. Geoff Cuvelier ${ }^{23}$. \\ Jignesh Dalal ${ }^{24}$. Magee L. DeFelice ${ }^{25} \cdot$ Kenneth B. DeSantes ${ }^{26}$. Lisa R. Forbes ${ }^{27}$ • Alfred Gillio ${ }^{28}$. Fred Goldman ${ }^{29}$.

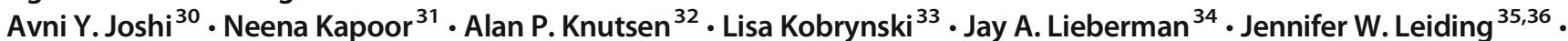 \\ Benjamin Oshrine ${ }^{35} \cdot$ Kiran P. Patel $^{37}$. Susan Prockop ${ }^{38}$ - Troy C. Quigg ${ }^{39} \cdot$ Ralph Quinones $^{40} \cdot$ Kirk R. Schultz $^{41}$. \\ Christine Seroogy ${ }^{42}$. David Shyr ${ }^{43,44}$. Subhadra Siegel ${ }^{45}$. Angela R. Smith ${ }^{46}$ - Troy R. Torgerson ${ }^{8}$. \\ Mark T. Vander Lugt ${ }^{47}$. Lolie C. Yu ${ }^{48}$. Morton J. Cowan ${ }^{1} \cdot$ Rebecca H. Buckley ${ }^{49}$. Christopher C. Dvorak ${ }^{1}$. \\ Linda M. Griffith ${ }^{50}$. Elie Haddad ${ }^{51}$. Donald B. Kohn ${ }^{52} \cdot$ Brent Logan $^{53} \cdot$ Luigi D. Notarangelo $^{54}$ • Sung-Yun Pai ${ }^{55,56}$. \\ Jennifer Puck ${ }^{1}$ • Michael A. Pulsipher ${ }^{31}$ • Jennifer Heimall ${ }^{57}$ (I)
}

Published online: 3 December 2020

(C) Springer Science+Business Media, LLC, part of Springer Nature 2020

Correction to: Journal of Clinical Immunology https://doi.org/10.1007/s10875-020-00865-9

The original article contained missing author. The revised author group and affiliations are shown above and below.

\section{Authorship Contributions}

- Morna Dorsey, Nicola AM Wright, Natalia S. Chaimowitz, Blachy J Dávila Saldaña, Holly Miller, Michael D. Keller, Monica S. Thakar, Ami J. Shah, MD designed data collection instruments, collected data, carried out the initial analyses, drafted the initial manuscript, and reviewed and revised the final manuscript.

- Morton J Cowan, Rebecca H. Buckley, Christopher C. Dvorak, Elie Haddad, Donald B. Kohn, Luigi D. Notarangelo, Sung Yun Pai, Jennifer Puck, MD, Michael Pulsipher, Jennifer Heimall conceptualized and designed the study, designed the data collection instruments, collected data, carried out the initial analyses, drafted the initial manuscript and reviewed and revised the manuscript.

Morna Dorsey and Nicola A. M. Wright contributed equally to this work.

The online version of the original article can be found at https://doi.org/ $10.1007 /$ s10875-020-00865-9

Jennifer Heimall

heimallj@email.chop.edu

Extended author information available on the last page of the article
- Linda Griffith and Brent Logan conceptualized and designed the study, designed the data collection instruments, carried out data analyses, and reviewed and revised the manuscript.

- Rolla Abu-Arja, Jeffrey Andolina, Victor Aquino, JL Barnum, Jeffrey J. Bednarski, Monica Bhatia, Francisco A. Bonilla, Manish J. Butte, Nancy J Bunin, Lauri M Burroughs, Sharat Chandra, Sonali Chaudhury, Karin Chen, Hey Chong, Geoff Cuvelier MD, FRCPC, Jignesh Dalal, Magee L. DeFelice, Kenneth B. DeSantes, Lisa R Forbes, Alfred Gillio, Fred Goldman, Avni Y Joshi, Neena Kapoor, MD, Alan P. Knutsen, MD, Lisa Kobrynski, Jay A Lieberman, Jennifer W Leiding, Benjamin Oshrine, Kiran P. Patel, Susan Prockop, Troy C. Quigg, Ralph Quinones, Kirk R. Schultz, Christine Seroogy, David Shyr, Subhadra Siegel, Angela R. Smith, Troy R. Torgerson, Mark T. Vander Lugt, Lolie $\mathrm{C}$ Yu collected data and critically reviewed the manuscript for important intellectual content.

- All authors approved the final manuscript as submitted and agree to be accountable for all aspects of the work.

Publisher's Note Springer Nature remains neutral with regard to jurisdictional claims in published maps and institutional affiliations. 


\section{Affiliations}

Morna Dorsey ${ }^{1}$ - Nicola A. M. Wright ${ }^{2} \cdot$ Natalia S. Chaimowitz $^{3} \cdot$ Blachy J. Dávila Saldaña $^{4,5} \cdot$ Holly Miller $^{6}$. Michael D. Keller ${ }^{7}$. Monica S. Thakar ${ }^{8} \cdot$ Ami J. Shah $^{9} \cdot$ Rolla Abu-Arja $^{10} \cdot$ Jeffrey Andolina $^{11}$ • Victor Aquino ${ }^{12}$. J. L. Barnum ${ }^{13}$. Jeffrey J. Bednarski ${ }^{14} \cdot$ Monica Bhatia $^{15} \cdot$ Francisco A. Bonilla $^{16} \cdot$ Manish J. Butte $^{17} \cdot$ Nancy J. Bunin $^{18}$. Lauri M. Burroughs ${ }^{8}$. Sharat Chandra ${ }^{19,20}$. Sonali Chaudhury ${ }^{21} \cdot$ Karin Chen $^{22} \cdot$ Hey Chong $^{13} \cdot$ Geoff Cuvelier $^{23}$. Jignesh Dalal ${ }^{24}$. Magee L. DeFelice ${ }^{25}$. Kenneth B. DeSantes ${ }^{26} \cdot$ Lisa R. Forbes $^{27}$ • Alfred Gillio ${ }^{28}$ • Fred Goldman $^{29}$.

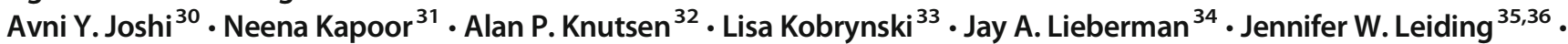
Benjamin Oshrine ${ }^{35}$. Kiran P. Patel ${ }^{37}$. Susan Prockop ${ }^{38}$. Troy C. Quigg ${ }^{39}$ - Ralph Quinones ${ }^{40} \cdot$ Kirk R. Schultz $^{41}$. Christine Seroogy ${ }^{42}$. David Shyr ${ }^{43,44}$. Subhadra Siegel ${ }^{45}$. Angela R. Smith ${ }^{46}$ - Troy R. Torgerson ${ }^{8}$.

Mark T. Vander Lugt ${ }^{47}$. Lolie C. Yu ${ }^{48}$. Morton J. Cowan ${ }^{1} \cdot$ Rebecca H. Buckley ${ }^{49}$. Christopher C. Dvorak ${ }^{1}$. Linda M. Griffith ${ }^{50}$. Elie Haddad ${ }^{51} \cdot$ Donald B. Kohn $^{52} \cdot$ Brent Logan $^{53} \cdot$ Luigi D. Notarangelo $^{54}$ - Sung-Yun Pai ${ }^{55,56}$. Jennifer Puck ${ }^{1}$ • Michael A. Pulsipher ${ }^{31}$ • Jennifer Heimall ${ }^{57}$ (D)

1 Division of Pediatric Allergy, Immunology, \& Bone Marrow Transplant, Benioff Children's Hospital, University of California San Francisco, San Francisco, CA, USA

2 Division of Hematology/Immunology, Department of Pediatrics, Alberta Children's Hospital, University of Calgary, Calgary, AB, Canada

3 Section of Immunology, Allergy and Retrovirology, Department of Pediatrics, William T. Shearer Center for Human Immunobiology, Texas Children's Hospital, Baylor College of Medicine, Houston, TX, USA

Division of Blood and Marrow Transplantation, Children's National Medical Center, Washington, DC, USA

5 Department of Pediatrics, George Washington University, Washington, DC, USA

6 Center for Cancer and Blood Disorders, Phoenix Children's Hospital, Phoenix, AZ, USA

7 Division of Allergy \& Immunology, Children's National Health System, and Division of Pediatrics, George Washington University, Washington, DC, USA

8 Fred Hutchinson Cancer Research Center, University of Washington, Seattle, WA, USA

9 Division of Stem Cell Transplantation and Regenerative Medicine, Lucille Packard Children's Hospital, Stanford School of Medicine, Stanford, CA, USA

10 Nationwide Children's Hospital, Columbus, OH, USA

11 Department of Pediatrics, Golisano Children's Hospital, University of Rochester Medical Center, Rochester, NY, USA

12 University of Texas, Southwestern, Dallas, TX, USA

13 UPMC Children's Hospital of Pittsburgh, Pittsburgh, PA, USA

14 Department of Pediatrics, Washington University in St. Louis School of Medicine, St. Louis, MO, USA

15 Pediatric Stem Cell Transplant Columbia, University Irving Medical Center, New York, NY, USA
16 Northeast Allergy, Asthma \& Immunology (private practice), Leominster, MA, USA

17 Division of Immunology, Allergy, and Rheumatology, Department of Pediatrics, University of California Los Angeles, Los Angeles, CA, USA

18 Cellular Therapy and Transplant Section, Division of Oncology, Children's Hospital of Philadelphia, Philadelphia, PA, USA

19 Division of Bone Marrow Transplantation and Immune Deficiency, Cincinnati Children's Hospital Medical Center, Cincinnati, OH, USA

20 Department of Pediatrics, University of Cincinnati College of Medicine, Cincinnati, OH, USA

21 Division of Pediatric Hematology, Oncology, Stem Cell Transplantation, Ann \& Robert H Lurie Children's Hospital of Chicago, Northwestern University Feinberg School of Medicine, Chicago, IL, USA

22 Division of Allergy and Immunology, Department of Pediatrics, University of Utah School of Medicine, Salt Lake City, UT, USA

23 Pediatric Blood and Marrow Transplant Program, CancerCare Manitoba, Department of Pediatrics and Child Health, Rady Faculty of Health Sciences, University of Manitoba, Winnipeg, MB, Canada

24 Pediatric Bone Marrow Transplant, Rainbow Babies and Children's Hospital, Cleveland, OH, USA

25 Division of Allergy and Immunology, Nemours/AI duPont Hospital for Children, Wilmington, DE, USA

26 Division of Hematology, Oncology and Bone Marrow Transplant, Department of Pediatrics, University of Wisconsin School of Medicine, Madison, WI, USA

27 William T Shearer Center for Human Immunobiology, Texas Children's Hospital, Baylor College of Medicine, Houston, TX, USA

28 Joseph M Sanzari's Childrens Hospital, Hackensack University Medical Center, Hackensack, NJ, USA 
29 Department of Pediatrics, Division of Hematology Oncology, University of Alabama at Birmingham, Birmingham, AL, USA

30 Pediatric and Adult Allergy/Immunology, Mayo Clinic, Rochester, MN, USA

31 Section of Transplantation and Cellular Therapy, Children's Hospital Los Angeles Cancer and Blood Diseases Institute, USC Keck School of Medicine, Los Angeles, CA, USA

Pediatric Allergy and Immunology, Cardinal Glennon Children's Hospital, St. Louis, MO, USA

33 Children's Healthcare of Atlanta, Emory University Department of Pediatrics, Allergy and Immunology, Atlanta, GA, USA

34 Department of Pediatrics, The University of Tennessee Health Science Center, Memphis, TN, USA

35 Division of Allergy and Immunology, Department of Pediatrics, University of South Florida, St. Petersburg, FL, USA

36 Johns Hopkins All Children's Hospital, Cancer and Blood Disorders Institute, St. Petersburg, FL, USA

37 Children's Healthcare of Atlanta, Atlanta, GA, USA

38 Department of Pediatrics, Bone Marrow Transplant Service, Memorial Sloan Kettering Cancer Center, New York, NY, USA

39 Pediatric Blood and Marrow Transplantation Program, Methodist Children's Hospital, San Antonio, TX, USA

40 Pediatric Hematology, Oncology and Bone Marrow Transplant, Children's Hospital Colorado, Aurora, CO, USA

41 Michael Cuccione Childhood Cancer Research Program, BC Children's Hospital and Research Institute, Vancouver, British Columbia, Canada

42 University of Wisconsin School of Medicine and Public Health, Madison, WI, USA

43 Division of Pediatric Hematology/Oncology, Department of Pediatrics, University of Utah School of Medicine, Primary Children's Hospital, Salt Lake City, UT, USA

44 Division of Stem Cell Transplant, Department of Pediatrics, Stanford Medicine, Lucile Packard Children's Hospital, Palo Alto, CA, USA
Division of Pediatric Pulmonology, Allergy and Immunology and Sleep Medicine, Westchester Medical Center, Valhalla, NY, USA

Pediatric Blood and Marrow Transplantation, University of Minnesota, Minneapolis, MN, USA

47 Blood and Marrow Transplant Program, University of Michigan, Ann Arbor, MI, USA

48 Division of Heme-Onc/HSCT, Children's Hospital/LSUHSC, New Orleans, LA, USA

49 Division of Allergy and Immunology, Department of Pediatrics and Department of Immunology, Duke University School of Medicine, Durham, NC, USA

50 Division of Allergy, Immunology and Transplantation, National Institute of Allergy and Infectious Diseases, National Institutes of Health, Bethesda, MD, USA

51 Pediatric Immunology and Rheumatology Division, CHU SainteJustine, Department of Pediatrics, Department of Microbiology, Immunology and Infectious Disease, University of Montreal, Montreal, QC, Canada

52 Department of Pediatrics, David Geffen School of Medicine, University of California, Los Angeles, Los Angeles, CA, USA

53 Division of Biostatistics, Medical College of Wisconsin, Milwaukee, WI, USA

54 Laboratory of Clinical Immunology and Microbiology, National Institute of Allergy and Infectious Diseases, National Institutes of Health, Bethesda, MD, USA

55 Division of Hematology-Oncology, Boston Children's Hospital, Boston, MA, USA

56 Department of Pediatric Oncology, Dana-Farber Cancer Institute, Boston, MA, USA

57 Division of Allergy and Immunology, Children's Hospital of Philadelphia, Wood 3301, 3401 Civic Center Blvd, Philadelphia, PA 19104, USA 